\title{
Statistical regimes of random laser fluctuations
}

\author{
Stefano Lepri,, , 7 Stefano Cavalieri, ${ }^{2,3}$ Gian-Luca Oppo, ${ }^{4}$ and Diederik S. Wiersma ${ }^{3,5}$ \\ ${ }^{1}$ Istituto dei Sistemi Complessi, Consiglio Nazionale delle Ricerche, \\ via Madonna del Piano 10, I-50019 Sesto Fiorentino, Italy \\ ${ }^{2}$ Dipartimento di Fisica, via G. Sansone 1 I-50019, Sesto Fiorentino, Italy \\ ${ }^{3}$ European Laboratory for Non-linear Spectroscopy, \\ via N. Carrara 1, I-50019 Sesto Fiorentino, Italy \\ ${ }^{4}$ SUPA and Department of Physics, University of Strathclyde, \\ 107 Rottenrow, Glasgow, G4 ONG, Scotland, U.K. \\ ${ }^{5}$ Istituto Nazionale per la Fisica della Materia, via G. Sansone 1 I-50019, Sesto Fiorentino, Italy
}

(Dated: November 7, 2006)

\begin{abstract}
Statistical fluctuations of the emitted light from random amplifying media are theoretically and numerically studied. The characteristic scales of the diffusive motion of photons lead to Gaussian or power-law (Lévy) distributed fluctuations depending on external control parameters. In the Lévy regime, the output pulse is highly irregular leading to huge deviations from a mean-field description. Monte Carlo simulations of a simplified model which includes the population of the medium demonstrate the two statistical regimes and provide a comparison with dynamical rate equations. Different statistics of fluctuations help to explain phenomenological observations reported in the experimental literature.
\end{abstract}

PACS numbers: 05.40.-a,42.65.Sf,42.55.Px

\section{INTRODUCTION}

Optical transport in disordered dielectric materials can be described as a multiple scattering process in which light waves are randomly scattered by a large number of separate elements. To first approximation this gives rise to a diffusion process. The propagation of light waves inside disordered dielectric systems shows several analogies with electron transport in conducting solids [1] and the transport of cold atom gasses [2]. A particularly interesting situation arises when optical gain is added to a random material. In such materials light is multiply scattered and also amplified. They can be realized, for instance, in the form of a suspension of micro particles with added laser dye or by grinding a laser crystal. Optical transport in such systems is described by a multiple scattering process with amplification.

If the total gain becomes larger then the losses, fluctuations grow and these systems exhibit a lasing threshold. The simplest form of lasing in random systems is based on diffusive feedback [3] where a diffusion process traps the light long enough inside the sample to reach an overall gain larger then the losses. Interference effects do not play a role in this form of random lasing. Diffusive random lasing has been observed in various random systems with gain, including powders, laser dye suspensions, and organic systems [4, [5, 6, 7, 8$]$. The behavior of such system shows several analogies with a regular laser, including gain narrowing, laser spiking and relaxation oscillations [5, 9]. Reports in literature of complex emission spectra from random lasers containing a collec-

*Electronic address: stefano.lepri@isc.cnr.it tion of spectrally narrow structures $10,11,12$ have triggered a debate about the possibility of lasing of Anderson localized modes in random systems 10]. Although Anderson localized modes can, in principle, form very interesting lasers resonators in a gain medium [13, 14], there has been no experimental evidence to date of independent random lasing and localization in the same (3D) random sample. In general, the observed spectra can be understood via a multiple scattering description based on the amplification of statistically rare long light paths that does not require localization or even interference [15]. These emission spectra exhibit a strongly chaotic behaviour, related to the statistical properties of the intensity above and around the laser threshold.

Theoretical descriptions of light transport in amplifying disordered media and random lasing have been based so far on a diffusive mechanism [9, 16, 17], using, for instance, a master-equation approach [18]. To accommodate the existence of localization related effects in the diffusion regime, theoretical 'anomalously localized states' have been proposed 19, 20, 21, 22, 23, 24]. Other attempts to describe random lasing include Monte Carlo simulations [25], finite difference time domain calculations 26], and an approach using random matrix theory [27]. A common feature of these studies is that the statistical properties of a disordered optical system change with the addition of optical gain. It was, for instance, proposed that such systems can exhibit Lévy type statistics in the distribution of intensities [28].

In this paper we report on a detailed study of the statistical fluctuations of the emitted light from random amplifying media, using both general theoretical arguments and results from numerical studies. We find that the characteristic scales of the diffusive motion of photons lead to Gaussian or power-law (Lévy) distributed fluc- 
tuations depending on external parameters. The Lévy regime is limited to a specific range of the gain length, and Gaussian statistics are recovered in the limit of both low and high gain. Monte Carlo simulations of a simplified model which includes the medium's population and parallel processing of a large number of random walkers, demonstrate the two statistical regimes and provide a comparison with dynamical rate equations.

In Section III we present some general arguments to explain the origin of the Lévy statistics in random amplifying media. In addition, we discuss the possibility of observing different statistical regimes. To check the validity of the general proposed scenario, we define a simple stochastic model that is suitable for numerical simulations (Section III). The rate equation corresponding to its mean-field limit are introduced in Section IV] The results of Monte Carlo simulations are presented in Section $\nabla$ and discussed with experimental results in the concluding Section.

\section{STATISTICS OF THE EMITTED LIGHT}

Let us consider a sample of optically active material where photons can propagate and diffuse. Our description is valid in the diffusive regime, hence we assume here that $\lambda$ is smaller of the photon mean free path $\ell, \lambda<\ell$. The origin of the Lévy statistics can be understood by means of the following reasoning. Spontaneously emitted photons are amplified within the active medium due to stimulated emission. Their emission energy is exponentially large in the path length $l$ i.e.

$$
I(l)=I_{0} \exp \left(l / \ell_{G}\right)
$$

where we have introduced the gain length $\ell_{G}$. On the other hand, the path length in a diffusing medium is a random variable with exponential probability distribution

$$
p(l)=\frac{\exp (-l /\langle l\rangle)}{\langle l\rangle}
$$

where $\langle l\rangle$ is the length of the photon path within the sample. The path length depends on both the geometry of the sample and the photon diffusion constant $D$. A simple estimate of $\langle l\rangle$ can be provided by noting that for a diffusive process with diffusion coefficient $D,\langle l\rangle$ is proportional to the mean first-passage time yielding [29]

$$
\langle l\rangle=\frac{v}{D \Lambda}
$$

where $v$ is the speed of light in the medium and $\Lambda$ is the smallest eigenvalue of the Laplacian in the active domain (with absorbing boundary conditions). For instance, $\Lambda=$ $q^{2}$ with $q=\pi / L$ for an infinite slab or a sphere with $L$ being the thickness or the radius, respectively [3].
The combination of Eqs. (11) and (2) immediately provides that the probability distribution of the emitted intensity follows a power-law

$$
p(I)=\frac{\ell_{G}}{\langle l\rangle} I^{-(1+\alpha)} \quad, \quad \alpha=\frac{\ell_{G}}{\langle l\rangle} .
$$

Obviously the heavy-tail (4) holds asymptotically and the distribution should be cut-off at small $I$. The properties of the Lévy distribution (more properly termed Lévy-stable) are well known [30]. The most striking one is that for $0<\alpha \leq 2$ the average $\langle I\rangle$ esists but the variance (and all higher-order moments) diverges. This has important consequences on the statistics of experimental measurements, yielding highly irreproducible data with lack of self-averaging of sample-to-sample fluctuations. On the contrary, for $\alpha>2$ the standard central-limit theorem holds, and fluctuations are Gaussian.

The gain length $\ell_{G}$ is basically controlled by the pumping energy i.e. by the population of the medium excitations. Increasing the latter, $\ell_{G}$ and the exponent $\alpha$ (see equation (4) ) decrease thus enhancing the fluctuations. At first glance, one can infer that the larger the pumping the strongest the effect. On the other hand, $\ell_{G}$ is a time-dependent quantity that should be determined selfconsistently from the dynamics. Indeed, above threshold the release of huge number of photons may lead to such a sizeable depletion of the population itself to force $\ell_{G}$ to increase. It can then be argued that when the depletion is large enough the Lévy fluctuation may hardly be detectable.

To put the above statements on a more quantitative ground we need to estimate the lifetime of the population as created by the pumping process. Following Ref. [3], we write the threshold condition as

$$
r=v / \ell_{G}-D \Lambda>0
$$

which is interpreted as "gain larger than losses", the latter being caused by the diffusive escape of light from the sample. Note that the condition $r=0$ along with Eqs. (3) and (4) implies $\alpha=1$ at the laser threshold.

For short pump pulses the time necessary to the intensity to become large is of the order of the inverse of the growth rate $r$. When this time is smaller than the average path duration within the sample $\langle l\rangle / v$, a sizeable amplification occurs on average for each sontaneously emitted photon leading to a strong depletion of the population. In this case we expect a Gaussian regime where a mean field description is valid. The conditions for the Lévy regime are $1 / r>\langle l\rangle / v$ and $\alpha \leq 2$ and can be written as:

$$
\frac{1}{2} \frac{v}{D \Lambda}<\ell_{G}<2 \frac{v}{D \Lambda} .
$$

Note that the lower bound of the above inequalities correspond to $\alpha=1 / 2$.

Without losing generality and for later convenience, let us focus on the case of a two-dimensional infinite slab of 
thickness $L$. In Fig. 1] we graphically summarize equation (6) by drawing a diagram in the $\left(L, \ell_{G}\right)$ plane. This representation is convenient since it allows to locate three different regions corresponding to different statistics. For convenience the line corresponding to the threshold $\alpha=1$ is also drawn. The three regions of statistical interest displayed in Fig. [1]are:

Subthreshold Lévy: weak emission with Lévy statistics with $1<\alpha<2$ (shaded region in Fig. 1] above the laser threshold line);

Suprathreshold Lévy: strong emission with Lévy statis-

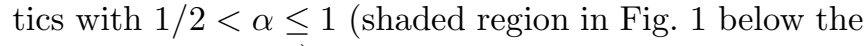
laser threshold line);

Gaussian: $\alpha<1 / 2$ strong emission with Gaussian statistics, $\alpha>2$ weak emission with Gaussian fluctuations.

Note that the first region corresponding to a nonlasing state, should display anomalous fluctuations as "precursors" of the transition. It should be also emphasized that the boundary between Lévy and Gaussian statistics is not expected to correspond to a sharp transition (as displayed in Fig. (1) but rather to a crossover region.

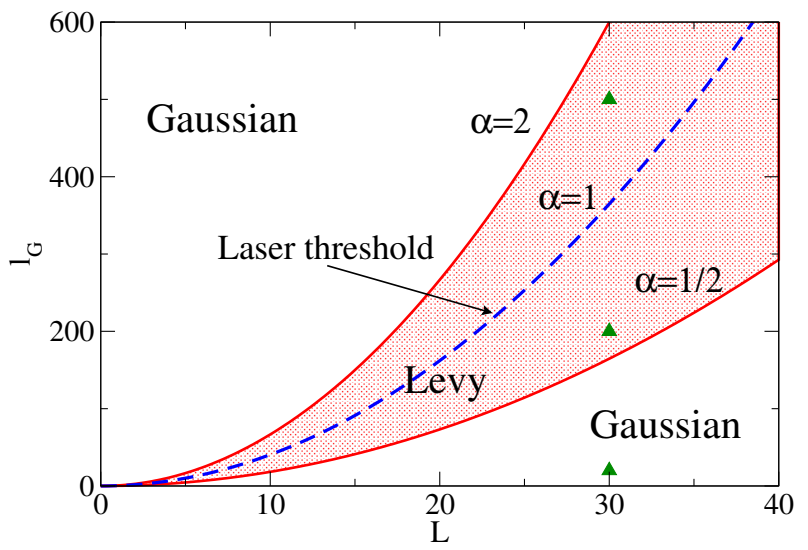

FIG. 1: (Color online) Different statistical regimes of fluctuations of a random laser with a two-dimensional slab geometry of height $L$. For comparison with the simulations reported below, all quantities are expressed in dimensionless units $\ell=1$, $v=1$. The symbols correspond to the parameter of Fig. 2

\section{NUMERICAL MODEL}

In order to provide evidence of the theoretical considerations presented in Section III we introduce a general, yet easy to simulate, model of random lasing. We consider a sample partitioned in cells of linear size $\ell$. Specifically, we deal with a portion of a two-dimensional square lattice. Thus the center of each cell is identified by the vector in$\operatorname{dex} \mathbf{r}=(x, y)$, with $x, y$ integers. In the following we will deal with a sample with a slab geometry i.e. $1 \leq x \leq L$, $1 \leq y \leq R L$. The total number of lattice sites is thus $R L^{2}$ where $R$ defines the slab aspect ratio. Periodic boundary conditions in the $y$ direction are assumed.
Within each cell we have the population $N(\mathbf{r}, t)$ of excitations. We consider an hypotetical three-level system with fast decay from the lowest laser level. If the population in the latter can be neglected we can identify $N$ as the number of atoms in the excited state of the lasing transition.

Isotropic diffusion of light is modeled as a standard random walk along the lattice sites. The natural time unit of the dynamics is thus given by $\Delta t=\ell / v$. We choose to describe the photon dynamics in terms of a set of $M$ walkers each carrying a given number of photons $n_{1}, \ldots n_{M}$. This may be visualized as an ensemble of "beams" propagating independently troughout the sample. Each of their intensities changes in time due to processes of stimulated and spontaneous emission. A basic description of those phenomena can be given in terms of a suitable master equation [18, 31] that would require to take into account the discrete nature of the variables. To further simplify the model we consider that the population and number of photons within each cell are so large for the evolution to be well approximated by the deterministic equation for their averages. In other words, the rate of radiative processes is much larger than that of the diffusive ones and a huge number of emissions occurs within the time scale $\Delta t$ [32]. With these simplifications $N$ and $n$ can be treated as continuous variables. Altogether the model is formulated by the following discretetime dynamics:

Step 0: pumping - The active medium is excited homogeneosly at the initial time i.e. $N(\mathbf{r}, 0)=N_{0}$. The value $N_{0}$ represents the the pumping level due to some external field. The initial number of walkers is set to $M=0$.

Step 1: spontaneous emission - At each time step and for every lattice site a spontaneous emission event randomly occurs with probability $\gamma N \Delta t$, where $\gamma$ denotes the spontaneous emission rate of the single atom. The local population is decreased by one:

$$
N \longrightarrow N-1
$$

and a new walker is started from the corresponding site with initial photon number $n=1$. The number of walkers $M$ is increased by one accordingly.

Step 2: diffusion - Parallel and asychronous update of the photons' positions is performed. Each walker moves with equal probability to one of its 4 nearest neighbours. If the boundaries $x=1, L$ of the system are reached, the walker is emitted and its photon number $n_{\text {out }}$ recorded in the output. The walker is then removed from the simulation and $M$ is diminished by one.

Step 3: stimulated emission - At each step, the photon numbers of each walker and population are updated deterministically according to the following rules:

$$
\begin{aligned}
& n_{i} \longrightarrow(1+\gamma \Delta t N) n_{i} \\
& N \longrightarrow\left(1-\gamma \Delta t n_{i}\right) N
\end{aligned}
$$

where $N$ is the population at the lattice site on which the $i$-th walker resides. 
Stochasticity is thus introduced in the model by both the randomness of spontaneous emission events (Step 1) and the diffusive process (Step 2). Note that the model in the above formulation does not include non-radiative decay mechanisms of the population. Furthermore, no dependence on the wavelength is, at present, accounted for; in general $\gamma=\gamma(\lambda)$.

The initialization described in Step 0 is a crude modelization of the pulsed pumping employed experimentally. It amounts to consider an infinitely short excitation during which the samples absorbs $N_{0}$ photons from the pump beam. As a further simplification we also assumed that the excitation is homogeneous on the whole sample. More realistic pumping mechanisms can be easily included in this type of modeling [33]. More importantly, as we are going to study the time dependence of the emission, this type of scheme applies to the case in which the time separation between subsequent pump pulses is much larger than the duration of the emitted pulse (i.e. no repumping effects are present).

Steps 1-3 are repeated up to a preassigned maximum number of iterations. The sum of all the photon numbers of walkers flowing out of the medium at each time step is recorded. The resulting time series is binned on a time window of duration $T_{W}$ to reconstruct the output pulse as it would be measured by an external photocounter. This insures that each point is a sum over a large number of events.

It should be emphasized that, although each walker evolves independenly from all the others, they all interact with the same population distribution, which, in turn, determines the photon number distributions. In spite of its simplicity, the model describes these two quantities in a self-consistent way.

For convenience, we choose to work henceforth in dimensionless units such that $v=1, \ell=1$ (and thus $\Delta t=1)$. The only independent parameters are then $\gamma$, the initial population $N_{0}$ (i.e. the pumping level) and the slab sizes $L, R L$.

\section{MEAN-FIELD EQUATIONS}

Before discussing the simulation of the stochastic model it is convenient to present some results on its mean-field limit. When both the population and photon number are large we expect the dynamics to be described by the rate equations for the macroscopic averages. This means that, up to relatively small fluctuations, the individual realization of the stochastic process should follow the solution of [3, 18]

$$
\begin{aligned}
& \dot{N}=-\gamma N(I+1) \\
& \dot{I}=D \Delta I+\gamma N(I+1)
\end{aligned}
$$

where $I(\mathbf{r}, t)$ is the number of photons in each cell, $\Delta$ denotes the two-dimensional discrete Laplacian and $D=$ $1 / 4$ in our case.
For simplicity, let us consider the case of a laterally infinite slab $(R \rightarrow \infty)$ in which both $N$ and $I$ depend on the $y$ coordinate, only. Absorbing boundary conditions are imposed, $I(0, t)=I(L+1, t)=0$. The integration is started from the same initial conditions of the stochastic simulations, namely $N(x, 0)=N_{0}, I(x, 0)=0$.

As a first remark, we note that the threshold condition (5) applies to (10) upon identifying

$$
\gamma N_{0}=\frac{1}{\ell_{G}} \quad, \quad q=\frac{\pi}{L+1} .
$$

We can thus define a critical value of the initial population $N_{c}=D q^{2} / \gamma$. For $N_{0}<N_{c}$ the total emission is very low being due to spontaneous processes that are only weakly amplified. On the contrary, for $N_{0}>N_{c}$ light amplification occurs: the number of photons within the sample increases exponentially in time at a rate given by (5), $r=\gamma N_{0}-D q^{2}=\gamma\left(N_{0}-N_{c}\right)$. After the pulse has reached a maximum and the population is depleted, the emission decreases strongly. An estimate of the decay time of the pulse is given by solving the linearized equations around the stationary state $N=0, I=0$. A straightfoward calculation yields that the long-time evolution is approximated by $N(x, t)=N_{q}(t) \sin (q x)$, $I(x, t)=I_{q}(t) \sin (q x)$ where

$$
\begin{aligned}
& N_{q}(t)=A \exp (-\gamma t) \\
& I_{q}(t)=A \frac{\gamma}{D q^{2}-\gamma} \exp (-\gamma t)+B \exp \left(-D q^{2} t\right),
\end{aligned}
$$

with $A, B$ being suitable time-independent amplitudes.

The above results have been checked by comparing them with the numerical solution of (10) obtained by simple integration methods of ordinary differential equations. In particular, we checked that both the rise and fall rates of the emission pulses (see the figures in the next section) are consistent with the expected values of $r$ and and Eqs. (13), respectively.

\section{MONTE CARLO SIMULATIONS}

In this Section we report the results of the simulation of the stochastic model. Preliminary runs were performed to check that lasing thresholds exist upon increasing of either the pumping parameter $N_{0}$ and the slab width $L$. The values are in agreement with the theoretical analysis presented above. In addition, checks of relations (2) and (3) have been performed.

As explained in Section III we monitored the outcoming flux (per unit length) $\phi$ as function of time. The results are compared with the corresponding quantity evaluated from Eqs. (10). In this case, $\phi$ is defined from the discrete continuity equation to be

$$
\phi=\frac{D}{2}[I(1, t)+I(L, t)] .
$$

The factor 2 comes from taking into account the contribution from the two boundaries $x=0, L$ of the lattice. 

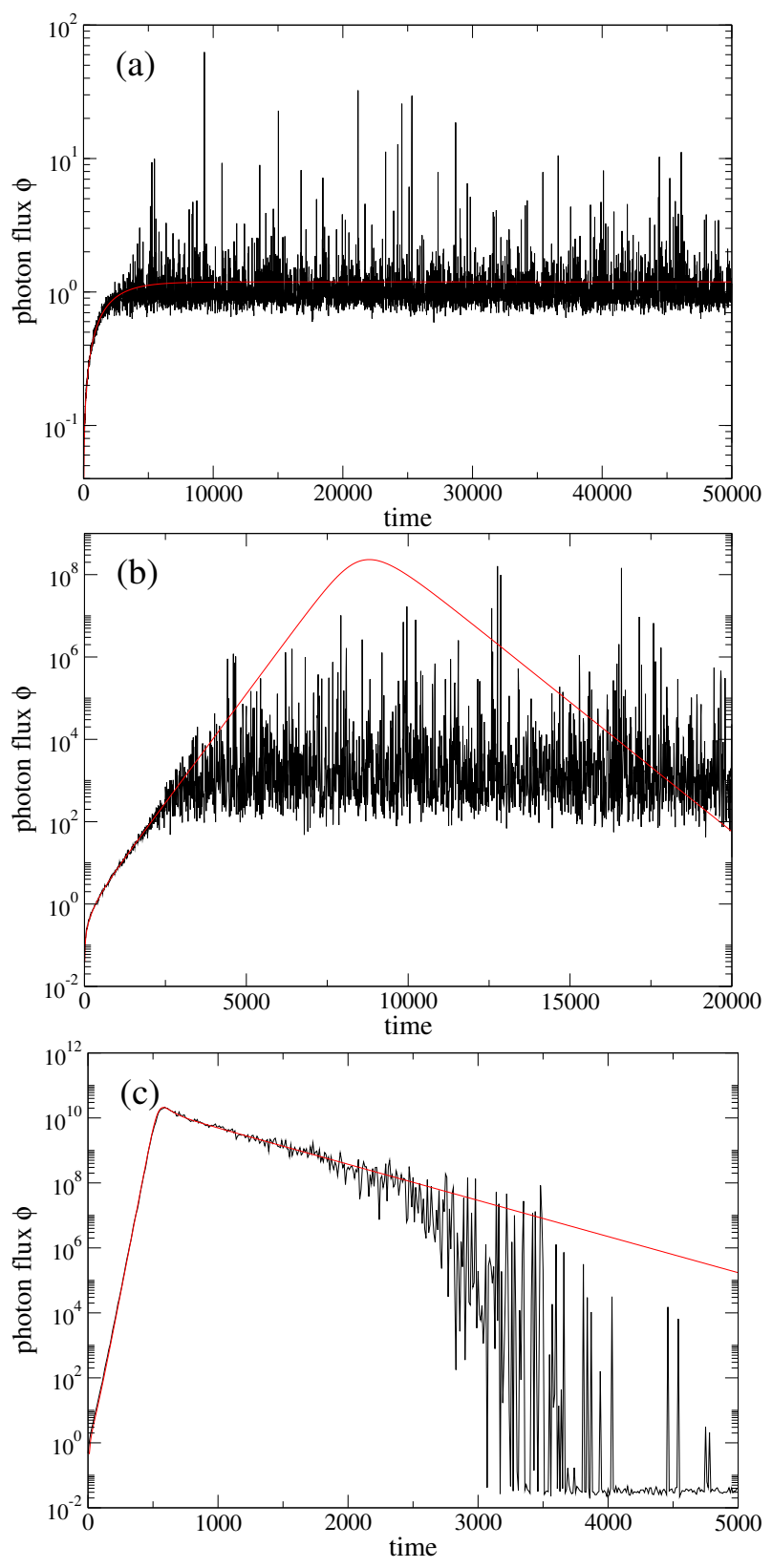

FIG. 2: (Color online) The photon flux (per unit length) as a function of time for a single shot and for $N_{0}=2 \times 10^{9}$, (a); $N_{0}=5 \times 10^{9}$ (b) and $N_{0}=50 \times 10^{9}$ (c). Smooth red lines are the mean-field results evaluated inserting the solutions of Eqs. (10) into (13). For both curves, data have been binned over consecutive time windows of duration $T_{W}=10$. Note the difference in the vertical-axis scales

The results of Monte Carlo simulation for a lattice with $L=30, R=20$ (18000 sites) and $\gamma=10^{-12}$ (yielding $N_{c}=2.5673 \times 10^{9}$ ) are reported in Fig. 2. The three chosen values of $N_{0}$ are representative of the three relevant statistical regions depicted in Fig. 1. they correspond to $\ell_{G}=500$ (Subthreshold Lévy), $\ell_{G}=200$ (Suprathreshold Lévy) and $\ell_{G}=20$ (Gaussian) respectively (see the triangles in Fig. (1). In the first two cases the total emission is highly irregular with huge departs from the expected mean-field behavior. Above the lasing threshold (Fig. П) single events ("lucky photons") may carry values of $n_{i}$ up to $10^{10}$. The resulting time-series are quite sensitive to inizialization of the random number generator used in the simulation. On the contrary, in the Gaussian case (Fig. 25) the pulse is pretty smooth and reproducible, except perhaps for its tails.

The evolution of the population $N$ displays similar features. We have chosen to monitor the volume-averaged population

$$
\frac{1}{R L^{2}} \sum_{\mathbf{r}} N(\mathbf{r}, t)
$$

normalized to its initial value for a better comparison. Fig. [3 shows the corresponding time-series for the same runs of Fig. 2 Again, large deviations from mean-field appear for the first two values of $N_{0}$. The inset shows that in correspondence with large-amplitude events the population abruptly decreases yielding a distinctive stepwise decay.

The non-smooth time decay is accompanied by irregular evolution in space. Indeed, a snapshot of $N(\mathbf{r}, t)$ reveals a highly inhomogeneous profile (see Fig. (4). Light regions are traces of high-energy events that locally deplete the population before exiting the sample.

For the Gaussian case (lowest curves in Fig. 2) similar considerations as those made for the corresponding pulse apply. Note that now the population level decays extremely fast. It reaches $10 \%$ of its initial value at $t \approx 600$ which is a factor two of the average residence time within the sample. This means that photons emitted after a few hundreds time steps have hardly any chance to be significantly amplified (i.e. $\ell_{G}$ has become too large).

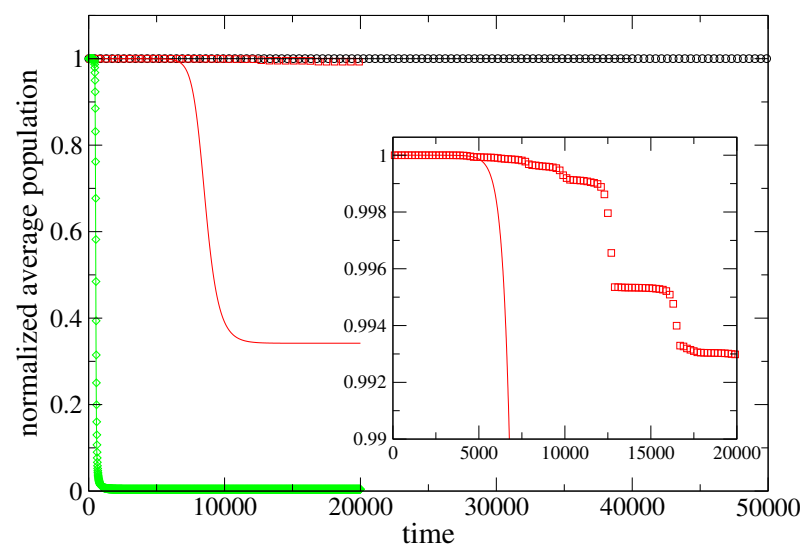

FIG. 3: (Color online) The normalized volume-averaged atomic population as a function of time for a single shot and for the same values of $N_{0}$ as in Fig. 2 Solid lines are the meanfield results evaluated inserting the solutions of Eqs. (10) into (14). The inset shows a magnification of the middle curves (case $N_{0}=5 \times 10^{9}$ of Fig. [2]). 


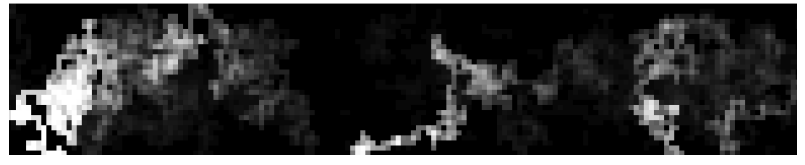

FIG. 4: A gray-scale plot of the atomic population distribution along a portion of the lattice for $t=10^{5}, N_{0}=5 \times 10^{9}$. White regions correspond to small values of $N$.

To check directly the validity of the power-law distribution (4) we computed the histograms of the photon number $n_{\text {out }}$ for each collected event. The result are given in Fig. [5 for three values of $N_{0}$ for which the Lévy distribution (4) is expected to occur. It should be recognized that this procedure is not entirely legitimate since we are dealing with a non-stationary process. This notwithstanding a clear power-law tail extending over several decades is observed. Note that the middle curve correspond to the threshold value $N_{0}=N_{c}$ for which we expect $\alpha=1$. Remarkably, the values of the exponents measured by fitting the data are in excellent agreement with the definition of $\alpha$ (see inset of Fig. 国). As predicted, no meaningful value smaller than $\alpha=1 / 2$ is obtained from the data.

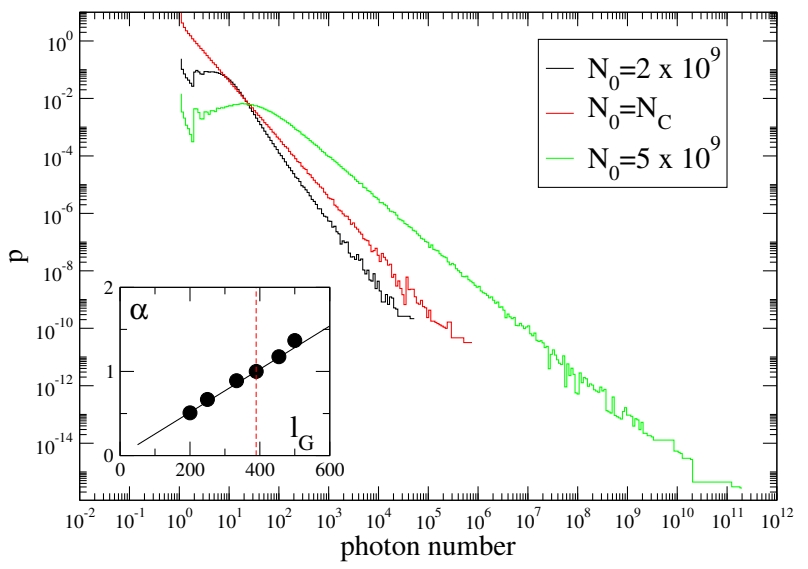

FIG. 5: (Color online) Histogram $p\left(n_{\text {out }}\right)$ of the emitted photon numbers $n_{\text {out }}$ for the two values of $N_{0}$ of Figs. 27a and b (lower and upper curves) and $N_{0}=N_{c}$ (middle). This middle curve has been vertically shifted for clarity. The inset reports the values of $\alpha$ obtained by power-law fit of the histograms as a function of the gain length $\ell_{G}$ as defined by Eq. (11). The thin solid line is the theoretical curve as computed by Eqs. (3) and (4). The dashed vertical line marks the lasing threshold.

Finally, to further elucidate the differences betweeen the two types of statistical regimes, we performed a series of simulations increasing the number of lattice sites. For comparison, we kept $L=30$ fixed and increase the aspect ratio $R$ up to a factor 4 . In this way, we increase the number of walkers accordingly. For the Gaussian case, we do observe the expected reduction of fluctua- tions around the mean-field solution. On the contrary, the wild fluctuations of the Lèvy case are hardly affected. This is a further confirmation of the scenario discussed in Section [I]

\section{DISCUSSION}

Based on heuristic arguments, we have shown in Section 1 that, depending on the value of the dimensionless parameter $D \Lambda \ell_{G} / v$, the fluctuations in the emission of a random laser subject to short pump pulses can be drastically different. In a parameter region extending both above and below threshold the photon number fluctuations follow a Lévy distribution thus displaying wild fluctuations and huge differences in the emission from pulse to pulse. In the suprathreshold case, such features have been indeed observed in experiments 28].

The exponent $\alpha$ of the Lévy distribution can be continously tuned upon changing the pumping level but it must be somehow bounded from below $(\alpha \gtrsim 1 / 2)$ as a further crossover to a Gaussian statistics is attained. Indeed, far above threshold, when the gain length is very small, a large and fast depletion of population occurs (saturation). This hinders the possibility of huge amplification of individual events. In this case all photons behave in a statistically similar way. As a consequence, the statistics is Gaussian and a mean-field description applies.

The above considerations have been substantiated by comparison with a simple stochastic model. It includes population dynamics in a self-consistent manner. In the Lévy regions, the simulation data strongly depart from the predictions of mean-field approximation due to the overwhelming role of individual rare events. As a consequence, the evolution of the population displays abrupt changes in time and is highly inhomogeneous in space.

To conclude this general discussion we remark that the width of the Lévy region as defined by inequalities (6) and depicted in Fig. 1 is of order $L^{2}$. Since in our simple model, $\ell_{G}$ is inversely proportional to the pump parameter (see equation (11)), the interval of $N_{0}$ values for which the Lévy fluctuations occur shrinks as $1 / L^{2}$. Therefore, the larger the lattice the closer to threshold one must be to observe them.

The existence of a such strong fluctuations is at variance with standard lasers that typically display them only at threshold. The emission statistics of random amplifying media has diverging moments in a finite region of parameters extending across the threshold curve.

The existence of different statistical regimes, their crossovers and their dependence on various external parameters enriches the possible experimental scenarios. Our theoretical work has shown that, depending on size, geometry, pumping protocols etc. the emission of random lasers may change considerably. This general conclusion should be a useful guidance in understanding past and future experiments on random amplifying media. 


\section{Acknowledgements}

We are indebted to R. Livi, S. Mujumdar, and A. Politi, for useful discussions and suggestions and to the Centro interdipartimentale per lo Studio delle Dinamiche Complesse (CSDC Università di Firenze) for hospitality. This work is part of the PRIN2004/5 projects Trans- port properties of classical and quantum systems and Silicon based photonic crystals funded by MIUR-Italy, and was financially also supported by LENS under EC contract RII3-CT-2003-506350, and by the EU through Network of Excellence Phoremost (IST-2-511616-NOE). GLO thanks SGI for kind support.
[1] P. Sheng, Introduction to Wave Scattering, Localization, and Mesoscopic Phenomena (Academic Press, San Diego, 1995).

[2] H. Gimperlein et al., Phys. Rev. Lett. 95, 170401 (2005); D. Clement et al., Phys. Rev. Lett. 95, 170409 (2005); C. Fort et al., Phys. Rev. Lett. 95, 170410 (2005).

[3] V.S. Letokhov, Zh. Éksp. Teor. Fiz. 53, 1442 (1967) [Sov. Phys. JETP 26, 835 (1968)].

[4] V.M. Markushev, V.F. Zolin, Ch.M. Briskina, Zh. Prikl. Spektrosk. 45, 847 (1986);

[5] C. Gouedard, et al., J. Opt. Soc. Am. B 10, 2358 (1993).

[6] N.M. Lawandy, et al., Nature (London) 368, 436 (1994); W.L. Sha, C.H. Liu, and R.R. Alfano, Opt. Lett. 19, 1922, (1994).

[7] M. Bahoura, K.J. Morris, and M.A. Noginov, Opt. Comm. 201, 405 (2002).

[8] D.S. Wiersma and S. Cavalieri, Nature 414, 708 (2001).

[9] D.S. Wiersma and A. Lagendijk, Phys. Rev. E 54, 4256 (1996); Light in strongly scattering and amplifying random systems, D.S. Wiersma (PhD thesis, Univ. of Amsterdam, 1995).

[10] H. Cao, Y. G. Zhao, S. T. Ho , E. W. Seelig, Q. H. Wang, and R. P. H. Chang, Phys. Rev. Lett. 82, 2278 (1999).

[11] H. Cao, J. Y. Xu, S.-H. Chang and S. T. Ho, Phys. Rev. E 61, 1985 (2000).

[12] R.C. Polson, M.E. Raikh, and Z.V. Vardeny, IEEE J. Sel. Topics in Q. Elec. 9, 120 (2003).

[13] P. Pradhan and N. Kumar, Phys. Rev. B 50, 9644 (1994).

[14] V. Milner and A.Z. Genack, Phys. Rev. Lett. 94, 073901 (2005)

[15] S. Mujumdar, M. Ricci, R. Torre, and D. S. Wiersma Phys. Rev. Lett. 93, 053903 (2004).

[16] A. Yu. Zyuzin, Europhys. Lett. 26, 517 (1994).

[17] S. John and G. Pang, Phys. Rev. A 54, 3642 (1996).
[18] L. Florescu and S. John, Phys. Rev. Lett. 93013602 (2004); Phys. Rev. E 69046603 (2004)

[19] B.L. Altshuler, V.E. Kravtsov, and I.V. Lerner, in Mesoscopic Phenomena in Solids, edited by B.L. Altshuler, P.A. Lee, and R.A. Webb (North- Holland, Amsterdam, 1991).

[20] A. D. Mirlin, Phys. Rep. 326, 259 (2000).

[21] M. Patra, Phys. Rev. E 67, 016603 (2003).

[22] V. G. Karpov, Phys. Rev. B 48, 4325 (1993).

[23] S. E. Skipetrov, and B. A. van Tiggelen, Phys. Rev. Lett. 92, 113901 (2004).

[24] V. M. Apalkov, M. E. Raikh, and B. Shapiro, Phys. Rev. Lett. 89, 126601 (2002)

[25] G. A. Berger, M. Kempe and A. Z. Genack, Phys. Rev. E 566118 (1997)

[26] X. Jiang and C.M. Soukoulis, Phys. Rev. Lett. 8570 (2000).

[27] C.W.J. Beenakker, Phys. Rev. Lett. 81, 1829 (1998).

[28] D. Sharma, H. Ramachandran and N. Kumar, Fluct. Noise Lett. 6 L95 (2006).

[29] S. Redner, A Guide to First-passage Processes, (Cambridge University Press, Cambridge, 2001).

[30] J.P. Bouchaud and A. Georges, Phys. Rep. 195, 127 (1990).

[31] H.J. Carmichael, Statistical Methods in Quantum Optics 1 (Springer-Verlag, Berlin, 1999).

[32] In our units this correspond to the condition $\gamma N n \ll 1$. For the parameters in the simulations $\gamma N \sim 10^{-3}$, i.e. the condition may be violated for short times. On the other hand this initial regime is irrelevant for the effects we are interested in.

[33] S. Mujumdar, S. Cavalieri and D.S. Wiersma, J. Opt. Soc. Am. B vol. 21201 (2004). 